\title{
SENSITIVITY OF SPACE STATION ALPHA JOINT ROBUST CONTROLLER TO STRUCTURAL MODAL PARAMETER VARIATIONS
}

Renjith R. Kumar, Paul A. Cooper, and Tae W. Lim

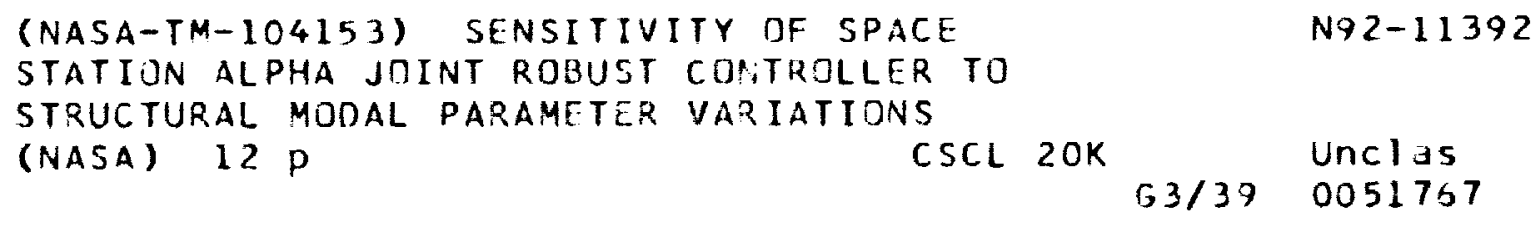

\section{NASก \\ National Aeronautics and \\ Space Administration}

Langley Research Center

Hampton, Virginia 23665 
. 


\title{
SENSITIVITY OF SPACE STATION ALPHA JOINT ROBUST CONTROLLER TO STRUCTURAL MODAL PARAMETER VARIATIONS
}

\author{
Renjith R. Kumar* \\ Analytical Mechanics Associates, Inc. \\ Hampton, Virginia \\ Paul A. Cooper** \\ NASA Langley Research Center \\ Hampton, Virginia \\ and \\ Tae W. $\mathrm{Lim}^{+}$ \\ Lockheed Engineering and Sciences Company \\ Hampton, Virginia
}

\begin{abstract}
The photovoltaic array sun tracking control system of - Space Station Freedom is described in this paper. A synthesis procedure for determining optimized values of the design variables of the control system is developed using a constrained optimization technique. The synthesis is performed to provide a given level of stability margin, to achieve the most responsive tracking performance, and to meet other design requirements. Performance of the baseline design, which is synthesized using predicted structural characteristics, is discussed and the sensitivity of the stability margin is examined for variations of the frequencies, mode shapes and damping ratios of dominant structural modes. The design provides enough robustness to tolerate a sizeable error in the predicted modal parameters. The paper concludes with an investigation on the sensitivity of performance indicators as the modal parameters of the dominant modes vary. The design variables are re-synthesized for varying modal parameters in order to achieve the most responsive tracking performance while satisfying the design requirements. This procedure of re--optimizing design parameters would be useful in improving the control system performance if accurate modal data are provided through an on-orbit modal identification experiment.
\end{abstract}

\section{Introduction}

To obtain electric power, Space Station Freedom (SSF) shown in Fig. 1 depends on photovoltaic (PV) solar arrays which track the sun during orbital daylight. The arrays are attached to deployable masts which are in turn attached through a rotary joint, called a Solar Beta Rotary Joint (Beta Joint), to the outboard portion of the transverse booms. The Beta Joint permits rotation of the arrays to compensate for the variation of the orbit plane with respect to the ecliptic plane. Rotary joints, called Solar Alpha Rotary Joints (SARJ or Alpha Joints), regulate the relative rotational position of the outboard

* Senior Project Engineer, Member AIAA.

** Senior Engineer, Associate Fellow AIAA.

+ Senior Engineer, Member AIAA. structure to the inboard structure. The attitude of the inboard structure is controlled by Control Moment Gyros (CMG's) and Reaction Control System (RCS) jets. The Alpha Joints are used to orient the array surface normal vectors along the solar vector so that maximum solar energy falls on the arrays during the daylight portion of each orbit. The Alpha Joint control is designed to be a basic position tracking system with minorloop velocity feedback to stabilize and provide damping to the rigid body tracking motion. A proportional-integral (P-I) compensation is added in both the velocity and position loops to minimize steady-state tracking error. 1

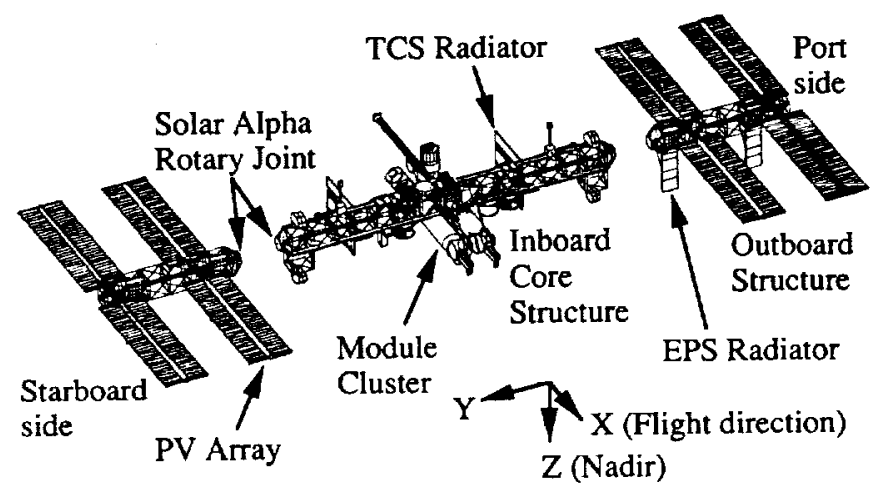

Fig. 1 SSF Assembly Complete configuration

The allowable rigid-body control bandwidth of the Alpha Joint controller encompasses the resonant structural frequencies of the outboard boom and photovoltaic system so that the possibility for adverse interaction between the control system and the dynamic response of the structure exists. To reduce the possible detrimental effect of control/structure interaction, a low-pass filter is added to the velocity loop to attenuate the structural response signal. The proper placement of the comer frequency of the filter and selection of values for gain setting of the P-I compensation in the velocity and position loops are required to provide optimum performance. The proper selection of these design values depends on the accuracy of the predictions of the structural frequencies and modal response at sensor locations.

The space station is too large and flexible to support its own weight on earth. Hence, the structural dynamic 
characteristics will have to be estimated from analytical models and component modal tests rather than from modal tests of the assembled structure. Further, the station is designed to support rcconfigurations for more power, payload installations and other activitics which can change the dynamic characteristics of the station. Because of the considerable uncertainty involved in predicting the dynamic characteristics of the station initially and over its lifetime, the sun tracking control system must be designed with a high degrec of stability robustness. This paper presents results from an investigation of the sensitivity of the control system stability margins to variations in modal parameters of dominant structural vibration modes. With this sensitivity established, the control system can be designed with a rcasonable degree of robustness to assure stable tracking for a given range of variation in structural parameters which might occur due to configuration changes and crtors in analytical estimation.

The paper describes the procedures to attenuate the possible control/structure interaction and 10 examine controller sensitivity to variations in structural modal parameters. First, the significant components of the space station related to the Alpha Joint control are described and the sun tracking control system is described. A baseline design is then determined using constrained optimization techniques to meet design requircments, to provide a given level of stability margin, and to obtain the most responsive tracking possible consistent with the assumed structural characteristics. Performance of the baseline design is discussed and the sensitivity of the stability margin is examined for variations of the natural frequencies, mode shape amplitudes and modal damping ratios of the dominant structural modes. The paper concludes with an investigation on the sensitivity of performance indicators as the modal parameters of the dominant modes vary. The design variables are re-synthesized for varying modal parameters in order to achieve the most responsive tracking performance while satisfying the design requirements.

\section{Description of space station and sun tracking function}

\section{Descriotion of space station structure}

The space station structure can be broadly divided into an inboard core structure and an outboard articulating structure. As shown in Fig. 1, the inboard core structure is comprised of a module cluster, center truss, thermal control system (TCS) radiators, and various user payloads. The port and starboard truss, PV arrays and electrical power system (EPS) radiators constitute the outboard articulating structure commonly referred to as the outboard structure. The attitude of the inboard core is maintained close to a local-vertical-local-horizontal (LVLH) orientation using active control devices such as the CMGs and/or RCS thrusters. The LVLH $\mathrm{X}$-axis is parallel to the flight direction, the LVLH Z-axis directed to the nadir, and the LVLH $\mathrm{Y}$-axis is orthogonal to the orbit plane. The power required for the space station is provided by the PV arrays as they track the sun. Due to the motion of the space station along its orbit, corc attitude fluctuations with respect to the LVLH orieniation, and the variations of the orbit geometry with respect to the sun, the oricntation of the PV arrays have to be constantly adjusted with respect to the inboard core to track the sun. This function is performed by the Alpha Joints and the Beta Joints. The Alpha Joints provide a relative rotational motion between the inboard core structure and the outboard articulating structure. The Beta Joints perform the PV array orientation adjustment with respect to the articulating outboard port and starboard truss.

Assuming that the station is maintained at an LVLH attitude, the Alpha Joint rotation rate would be the orbital rate, completing a revolution every 90 minutes. The Beta Joint rotation is extremely slow over an orbit and follows closely the yearly variation of the orbit plane. Therefore, the Beta Joint drive and control is not addressed in this paper.

\section{Bhysical description of Alpha Joint}

Each Alpha Joint consists of dual motors, dual resolvers, a motor controller, drive pinions, a bull gear and trundle bearings as depicted schematically in Fig. 2.2 The motor provides the torque required to rotate the outboard structure. The amount of control torque provided by the motor is determined by the motor controller based on the measurements obtained by the joint resolver and the desired rotation data from the Velocity Vector Generator (VVG) located on the inboard structure. The motor drive pinion to bull gear ratio has been carefully selected to minimize mechanical parts count and hence maximize reliability. ${ }^{3}$ The bull gear is rigidly attached to the outboard structure through a shear plate. The large bull gear (about 10 fect in diameter) is equipped with trundle bearings in order to

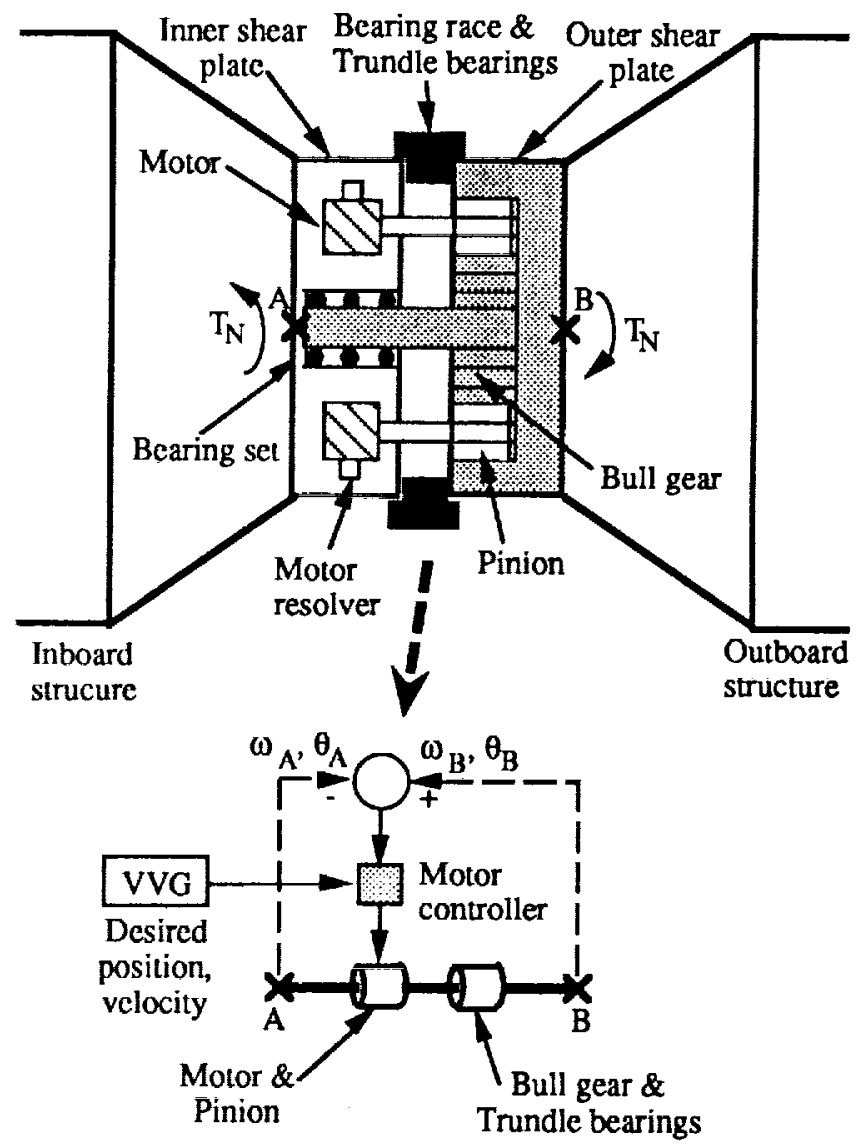

Fig. 2 Schematic of Alpha Joint drive train and control system 


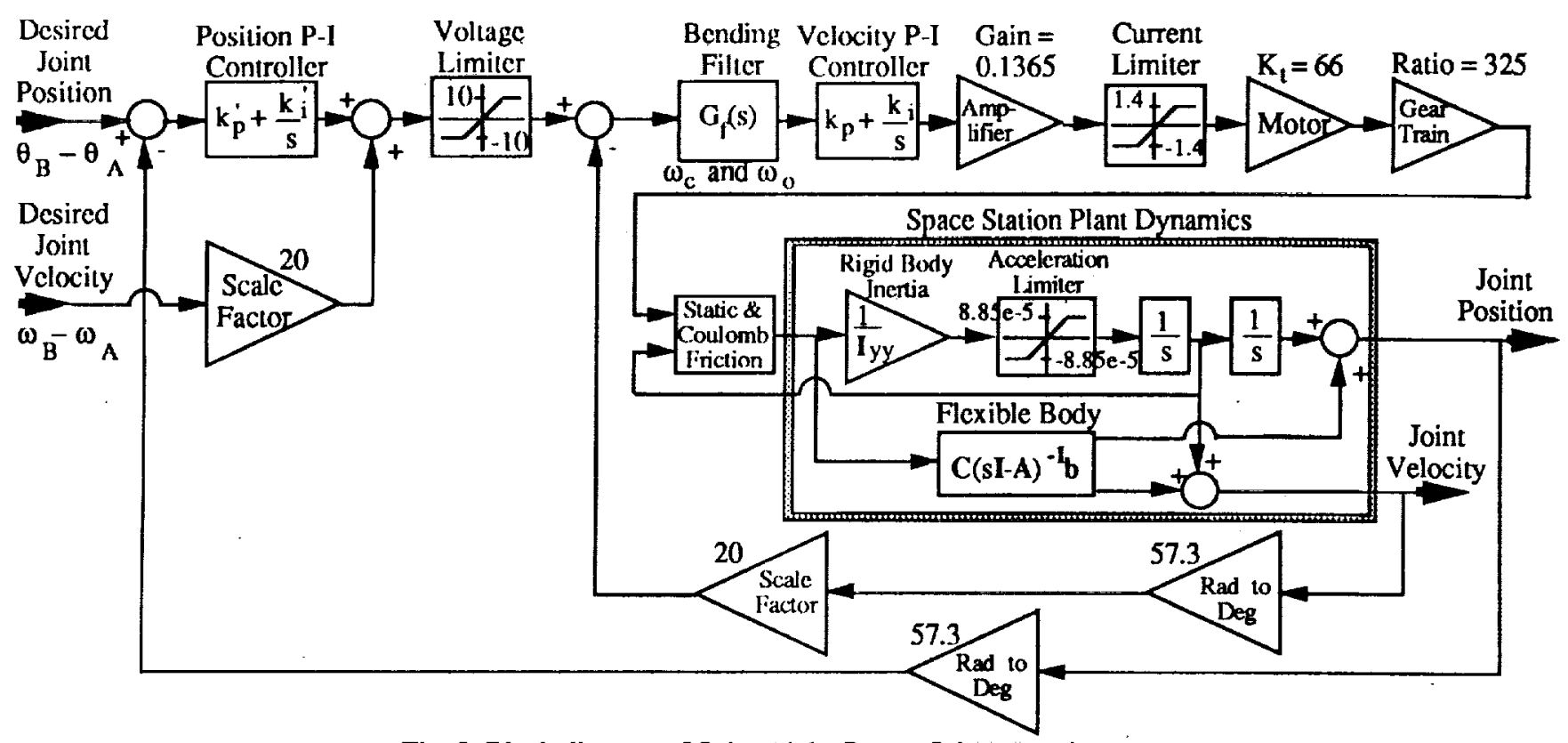

Fig. 3 Block diagram of Solar Alpha Rotary Joint control system

accommodate large temperature gradients. The trundle bearings are the main source of friction. A sel of high power roll rings (not shown in Fig. 2) carries power across the joint as the joint rotates. $^{3}$

\section{Description of Alpha Joint control system}

The SARJ motor controller generates the required motor torque based on the difference between the desired and measured relative joint angular position and angular velocity at points $A$ and $B$ as shown in Fig. 2. Point $A$ is fixed to the inboard structure while point $B$ is located on the outboard structure. The desired relative joint velocity command is determincd by the VVG located on the inboard structure and is an input to the SARJ motor control system. As the SARJ position leads or lags the desired position (also provided by the VVG), the input to the velocityloop is increased or decreased to compensate for the position error. Hence the reference input to the control system includes the desired relative angular velocity $\left(\omega_{B}-\omega_{A}\right)$ and the desired relative angular position $\left(\boldsymbol{\theta}_{\mathrm{B}}-\boldsymbol{\theta}_{\mathrm{A}}\right)$.

The detailed control system used in this paper is based on the design obtained from the SSF Preliminary Design Revicw document. ${ }^{4}$ A block diagram of the control system is shown in Fig. 3. The control system consists of an inner velocity servo loop which tracks the desired joint velocity and an outer position servo loop which increases or decreases the velocity command depending upon the position error. The velocity command is converted to a voltage command and the maximum allowable SARJ velocity is maintained by a voltage limiter.

The inner servo loop includes a fourth order Butterworth bending filter ${ }^{5}$ with two additional zeros to roll-off the high frequency structural modes. The two additional zeros are included to reduce the loss of rigid-body phase margin due to phase shift. The transfer function of the bending filter is

$$
G_{f}(s)=\frac{\omega_{c}^{4}\left(s^{2} / \omega_{o}^{2}+1.4 s / \omega_{o}+1\right)}{s^{4}+2.6 \omega_{c} s^{3}+3.4 \omega_{c}^{2} s^{2}+2.6 \omega_{c}^{3} s+\omega_{c}^{4}}
$$

where the comer frequency $\left(\omega_{\mathrm{c}}\right)$ and the frequency of the zeros $\left(\omega_{0}\right)$ are design parameters.

While the inner velocity feedback loop increases system damping, it also increases steady-state tracking error. Integral control helps to reduce steady state errors. Therefore, a proportional-integral (P-I) controller is used for the inner velocity loop and a similar P-I controller is used for the outer position loop. The position loop has a double integrator (one in the outer loop and the other in the inner loop) to track a ramp signal with zero steady state error. The P-I controllers are also provided with integration limits to prevent the system from being overdriven. ${ }^{4}$ This is required due to the acceleration limits imposed on the SARJ. Each P-I controller has two gain settings: $k_{p}$ and $k_{i}$ are the proportional and integral gains for the velocity loop and $\mathrm{k}_{\mathrm{p}}^{\prime}$ and $\mathrm{k}_{\mathrm{i}}^{\prime}$ are the corresponding gains for the position loop. These are also included as design variables during the synthesis of the control system design.

The power amplifier shown in Fig. 3 is accompanied by a current limiter. A 66 in-lbf/amp motor torque constant and a gear ratio of 325 is assumed. ${ }^{4}$ The output torque from the gear is subject to the large static and dynamic friction of the trundle bearings. The output torque must exceed the static friction of $3580 \mathrm{in}-\mathrm{lbf}$ to initiate motion of the SARJ. Once the motion is initiated, a net torque, which exceeds the dynamic friction of $2870 \mathrm{in}-\mathrm{lb}$, is applied to the structure at point B (actuator point) as shown in Fig. 2.

A finite-element model of the space station structure is used to compute the natural frequencies and mode shapes. A flexible body state space model of the structure is formed from the modal data. This is combined with the rigid body model of the outboard siructure to characterize the dynamics of the space 
station structure. The rigid body inertia of of the outboard structure (either port or starboard side) about the $\mathrm{Y}$-axis $\left(I_{Y Y}\right)$ is $1.75 \times 10^{7} \mathrm{lbf}-\mathrm{in}-\mathrm{sec}^{2}$. Details of the finite-element model and modal description are given in the next section. The net torque $\left(\mathrm{T}_{\mathrm{N}}\right.$ ) applied to the structure at actuator point B (Fig. 2) causes motion of the flexible structure. The net torque applied on the outboard structure causes a reaction torque on the core structure. This reaction torque has to be compensated by RCS jet and/or CMG torques to maintain the attitude of the core structure. This paper assumes that the reaction torque is compensated ideally and the iigid body attitude of the core structure remains stationary. The joint position and velocity containing both rigid body and elastic components are measured and fed back to close the control system loops.

\section{Dynamic characteristics of Space Station Freedom}

A finite element model of SSF was used to investigate the influence of clastic response to the Alpha Joint control system performance. To provide a rotational degree-of-freedom (DOF) about the $\mathrm{Y}$-axis of the outboard structure, two coincident grids were placed at the center node of the Alpha Joint as indicated in Fig. 4. These grids are rigidly connected in the other five DOF. The undamped natural frequencies of the finite-element model are shown in Fig. 5. For the model used, there are 240 modes below $5 \mathrm{~Hz}$ including the eight rigid body modes.

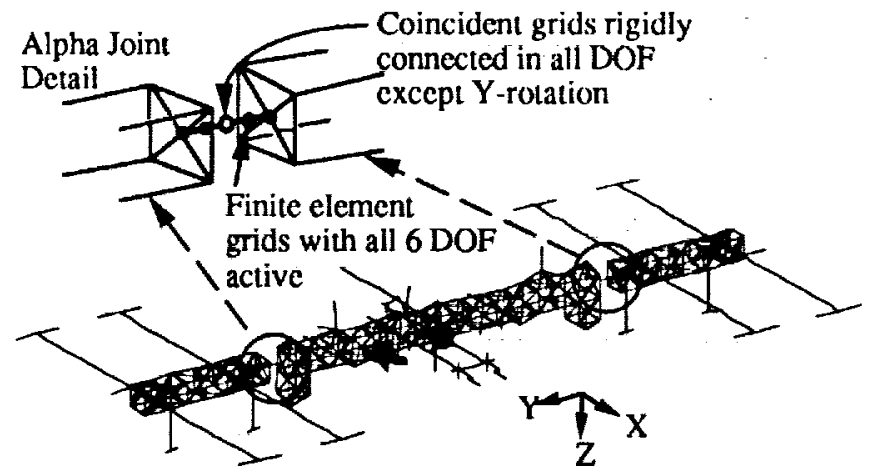

Fig. 4 Finite element model of Space Station Freedom

Since the outboard structure is subject to a continuous rotational motion while the SARJ is actively controlled, the influence of the outboard structure orientation on the SSF dynamic characteristics was investigated. The outboard structure was rotated 90 degrees from the minimum drag PV oricntation shown in Fig. 4 and the natural frequencies and mode shapes were calculated. The resulting maximum drag PV orientation SSF cxhibits a natural frequency distribution almost identical to that of the minimum drāg orientation. Also, the transfer function characteristics between the two points where the SARJ controller is applied (points A and B in Fig. 2) were almost identical. Thus the dynamics of SSF with respect to the SARJ control is considered to be time invariant for this study. Moreover, due to the symmetry of the space station structure, the dynamic characteristics of the SARJ at the port and starboard sides are nearly identical. Thus, as a representative plant model, the port SARJ with a minimum drag PV array orientation is employed in this paper.

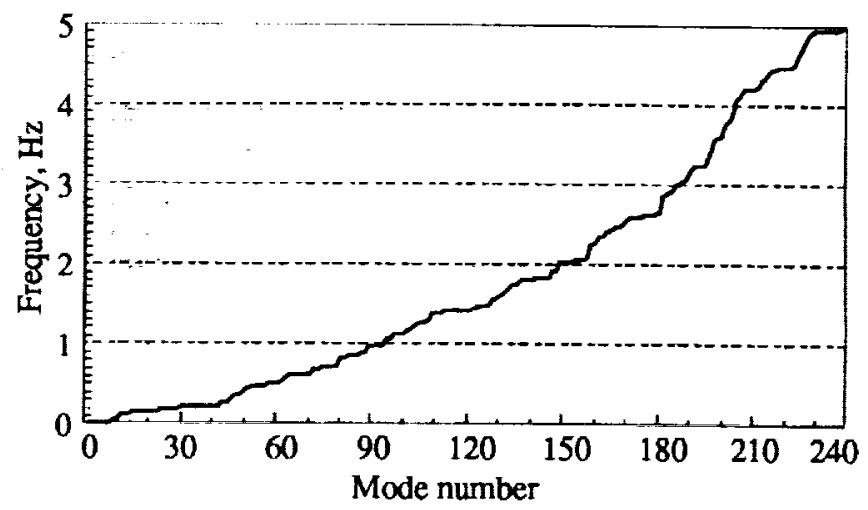

Fig. 5 Undamped natural frequencies of SSF below $5 \mathrm{~Hz}$

The equation of motion governing the flexible response at the port SARJ is represented by

$$
\begin{aligned}
& \dot{\mathbf{x}}=A \mathbf{x}+b u \\
& \mathbf{y}=\mathbf{C x}
\end{aligned}
$$

where $u$ is the control torque and

$$
\begin{aligned}
& x=\left\{\begin{array}{c}
\mathbf{q} \\
\dot{q}
\end{array}\right\}, A=\left[\begin{array}{cc}
0 & I \\
-\Omega^{2} & -2 Z \Omega
\end{array}\right], b=\left\{\begin{array}{c}
0 \\
T \\
\phi_{B}
\end{array}\right\} \\
& y=\left\{\begin{array}{c}
\theta_{B}-\theta_{A} \\
\omega_{B}-\omega_{A}
\end{array}\right\} \text {, and } C=\left[\begin{array}{cc}
\phi_{B}-\phi_{A} & 0 \\
0 & \phi_{B}-\phi_{A}
\end{array}\right]
\end{aligned}
$$

where $q$ is the modal displacement vector; $\theta_{A}$ and $\theta_{B}$ are the angular displacements at the points $A$ and $B$, respectively; $\omega_{A}$ and $\omega_{B}$ are the angular velocities at the points $A$ and $B$, respectively; $\Omega=\operatorname{diag}\left\{\omega_{i}\right]$ and $Z=\operatorname{diag}\left[\zeta_{i}\right\}$ in which $\omega_{i}$ and $\zeta_{i}$ are the natural frequency and modal damping ratio of the ith mode, respectively; $\phi_{A}$ and $\phi_{B}$ are the row vectors of the unity mass normalized mode shape matrix corresponding to the $Y$ rotational DOF at the points $A$ and $B$, respectively. Modal damping ratio of $0.1 \%$ for all the flexible modes is assumed as a baseline value for the design and simulation of the SARJ control system.

\section{Synthesis orocedure of control system design variables}

\section{Control system requirements}

The SARJ control system discussed earlier has six design variables whose values can be adjusted to optimize control system performance and to satisfy prescribed requirements. The control system requirements can be classified as frequency-domain and time-domain requirements. The frequency domain requirements in both inner and outer loops are as follows: (1) Rigid-body open-loop gain and phase margins (GM and PM) must be greater than or cqual to $6 \mathrm{~dB}$ and 45 degrees, respectively, to assure a stable rigid-body motion; (2) Apparent gain margin (AGM), defined as the minimum distance of the open-loop gain from the zero $\mathrm{dB}$ line in the frequency range encompassing the structural resonance frequencies, should be at least $20 \mathrm{~dB}$ to guarantee 
sufficient stability robustness to uncertainties in the predicted modal parameters; and (3) The closed-loop poles associated with rigid body and controller should have a minimum damping ratio of 0.5. By constraining rigid body and controller closed-loop poles to a prescribed sector in the complex plane, this frequencydomain constraint assures low overshoot during the transient response. The time-domain requirements ${ }^{4}$ include small steadystate pointing errors, small peak-to-pcak variation of stcady-statc pointing errors over long time intervals and low jitter. The jitter is defined as the peak-to-peak variation of the position error in one second.

The Preliminary Design Review document ${ }^{4}$ requires that the inner and outer closed-loop bandwidths $\left(\mathrm{BW}_{\mathrm{v}}\right.$ and $\mathrm{BW}$ ) be between 0.01 to $1 \mathrm{~Hz}$. This requirement is treated in this paper as part of a performance index which is to be maximized. Bandwidth is a measure of the responsiveness of a control system (closcly rclated to the rise time) and also represents a disturbance rejection threshold. Core attitude fluctuations and the maneuvers for feathering and debris collision avoidance may demand large atitude rate changes and thus motivate higher responsiveness of the control system. The other component of the performance index to be maximized is the magnitude of the real part of the dominant rigid-body and controller closed-loop pole $(\sigma)$. The dominant pole is defined here as the rigid-body and controller closed-loop pole (for both inner and outer loops) closest to the imaginary axis. This is equivalent to minimizing a settling time in a time-domain analysis. Table 1 summarizes the design objective, the control system frequency-domain and time-domain requirements, and the design variables.

Table 1 Summary of Alpha Joint controller design objective, variables, and constraints

\begin{tabular}{|c|c|}
\hline $\begin{array}{c}\text { Design } \\
\text { objective }\end{array}$ & $\begin{array}{l}\text { Maximize position and velocity loop bandwidth } \\
\text { and minimize settling time }\end{array}$ \\
\hline $\begin{array}{c}\text { Design } \\
\text { variables }\end{array}$ & $\begin{array}{l}\text { Controller gains }\left(k_{p}, k_{i}, k_{p}^{\prime} \text { and } k_{i}^{\prime}\right) \\
\text { Compensation filter brcak frequencies }\left(\omega_{c} \text { and } \omega_{\alpha}\right)\end{array}$ \\
\hline Consuraints & $\begin{array}{l}\text { Rigid-body gain margins } \geq 6 \mathrm{~dB} \\
\text { Rigid-body phase margins } \geq 45 \mathrm{deg} \\
\text { Apparent gain margins in structural resonant } \\
\text { frequency range } \geq 20 \mathrm{~dB} \\
\text { Minimum rigid body and controller } \\
\text { damping ratio } \geq 0.5 \\
\text { Bandwidth: between } 0.01 \text { and } 1 \mathrm{~Hz} \\
\text { Steady-state pointing error } \leq 0.58 \mathrm{deg} \\
\text { Peak-to-peak variation of steady-state pointing } \\
\text { error over } 30 \text { min: between } \pm 0.5 \mathrm{deg} \\
\text { Jitter } \leq 0.01 \mathrm{deg} / \mathrm{sec}\end{array}$ \\
\hline
\end{tabular}

\section{Synthesis orocedure}

The plant model includes the rigid body incria of the outboard structure about the Alpha Joint axis and the flexible modes of the entire structure. Figure 6 shows a frequency response function (FRF) of the rigid plant only compared with the FRF of the rigid body with all flexible modes of the finite element model up to a frequency of $5 \mathrm{~Hz}$. The FRF shown is the magnitude ratio of the velocity response to net torque applied and is plotted using a decibel scale against the log of the frequency. A control system designed with only the rigid plant taken into accouni could become unstable if the dominant flexible modes are not well attenuated by the control system. The dominant flexible modes (or dominant modes) are the most influential modes among the flexible modes in determining the apparent gain margin. The flexible modes are most likely to be influential if their gain is high and their frequency is low. Figure 6 indicates that the dominant flexible modes occur at frequencies of $0.485 \mathrm{~Hz}$ and $0.486 \mathrm{~Hz}$. The corresponding mode shapes are shown in Fig. 7. These modes correspond to a rigid body rotation of the outboard trusses coupled with symmetric and anti-symmetric bending of the PV arrays while most parts of the inboard structure remain stationary. Other modes which might interfere with rigid body controllers are at higher frequencies. Even though their gains might be higher than the modes selected as dominant, their influence would be attenuated further by a lowpass filter used to roll-off the effects of the dominant modes and can hence be ignored during the control system design.

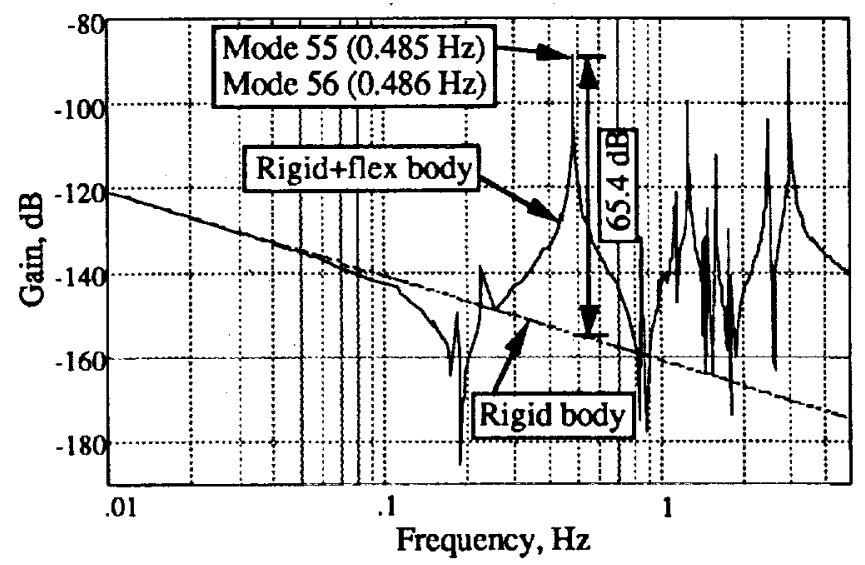

Fig. 6 Frequency response of rigid body and rigid+flex body plants (velocity response to control torque)

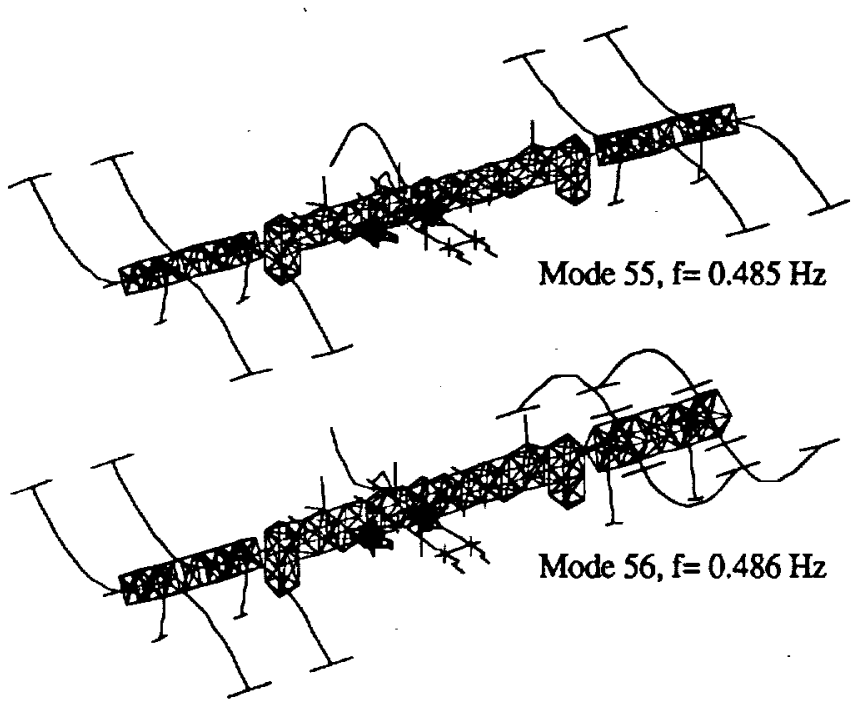

Fig. 7 Dominant modes for SSF Alpha Joint control

It is desirable that all the flexible modes be removed from the plant during the synthesis of design variables to ease the computational load. The dominant flexible modes have a gain of approximately $65.4 \mathrm{~dB}$ above the rigid body gain as shown in Fig. 6 . Hence the $20 \mathrm{~dB}$ apparent gain margin constraint for the plant 
with the nexible modes is equivalent to a constraint of $85.4 \mathrm{~dB}$ apparent gain margin for the rigid body plant at the frequency of $0.49 \mathrm{~Hz}$. For the synthesis of design variables, the plant is considered as a rigid body with one of the constraints modified as described above. This consideration is used only for design purposes and not for subsequent frequency response analyses or lime response simulations.

To further simplify the synthesis procedure, the limiters in the control system are ignored. The friction block is replaced by a transfer function of unity, which is a conservative assumption for a robust design. The linearized plant and control system are transformed to the frequency domain and the design symthesis is performed using a constrained optimization scheme.

Now, the synthesis problem can be stated as follows: Find the optimum values for the six design variables $\left(\omega_{c}, \omega_{0}, k_{p}, k_{i}, k_{p}^{\prime}\right.$ and $\left.k_{i}^{\prime}\right)$ which maximize the performance index

$$
J=\mu_{1} B W_{v}+\mu_{2} B W_{p}+\mu_{3} \sigma
$$

while satisfying the following constraints:

Velocily-loop rigid-body gain margin $\geq 6 \mathrm{~dB}$

Position-loop rigid-body gain margin $\geq 6 \mathrm{~dB}$

Velocity-loop rigid-body phasc margin $\geq 45$ degrees

Position-loop rigid-body phase margin $\geq 45$ degrees

Velocity-loop rigid-body gain at the frequency of $0.49 \mathrm{~Hz} \leq-85.4 \mathrm{~dB}$

Minimum rigid-body position and velocity closed-loop damping factor $\geq 0.5$.

The scalars $\mu_{1}, \mu_{2}$ and $\mu_{3}$ are weighting factors selected to give equal weights to each element of the performance index. Equal weights of unity are used for $\mu_{1}$ and $\mu_{2}$ since the velocity and position closed-loop bandwidths are of the same magnitude. The magnitude of $\sigma$ is an order of magnitude less than the bandwidths expressed in rad/sec. To give approximately cqual importance to the settling time, $\mu_{3}=10$ is selected. The control torque is not incorporated in the synthesis problem either as a performance index or as a constraint. It is assumed that the available control torque will be sufficient to perform the maneuver required by the synthesized design.

The constrained optimization problem is solved using a nonlinear programming method available in MATRIXX software. ${ }^{6}$ A brief description of the method is given here. The constrained nonlinear optimization problem is initially approximated by a linearly constrained optimization problem with an augmented Lagrangian objective function. Then, scquential quadratic programming is implemented to solve the optimization problem with the new objective function approximated to the second order. The software uses a recent extension ${ }^{7}$ of the Karmarkar's interior point algorithm to solve the resulting quadratic programming problem. It was noticed that there existed more than one local minimum for the optimization problem so that selection of the initial guess values for the six design variables was important in obtaining the "best" local minimum. However, one cannot be guaranteed that this "best" local minimum obtained is the global minimum.

The resulting optimized values of the design variables with pcrformance index and constraints are summarized in Table 2. The design satisfies all the prescribed design requirements. The steady-state time domain requirements listed in Table 1, which were not enforced during the optimization, were checked for violation using the optimized design variables through time response simulation. The closed-loop poles of the velocity and position loops were inspected to ensure stability. The following section discusses the design results in detail.

\section{Design results}

The results of the design are summarized in Table 2 . The bandwidths of the velocity and position loops are within the range specified in the space station program requirements. Design constraints are all met near or at the boundary of the constraints. The compensated Bode plot of the velocity loop on the port side

Table 2 Results of Alpha Joint controller design

\begin{tabular}{|c|c|c|c|}
\hline & Description & Requirements & Optimized results \\
\hline $\begin{array}{l}\text { Design } \\
\text { objectives }\end{array}$ & $\begin{array}{l}\text { Velocity-loop bandwidth (BWv) } \\
\text { Position-loop bandwidth (BWp) } \\
\text { Distance of dominant pole to imaginary axis }\end{array}$ & $\begin{aligned} & 0.01 \mathrm{~Hz} \leq \mathrm{BWv} \leq 1 \mathrm{~Hz} \\
& 0.01 \mathrm{~Hz} \leq \mathrm{BWp} \leq 1 \mathrm{~Hz} \\
& \text { N/A }\end{aligned}$ & $\begin{array}{c}0.053 \mathrm{~Hz} \\
0.027 \mathrm{~Hz} \\
0.026\end{array}$ \\
\hline \multirow{6}{*}{$\begin{array}{l}\text { Design } \\
\text { variables }\end{array}$} & \multirow{6}{*}{$\begin{array}{l}k_{\mathrm{p}} \\
\mathbf{k}_{\mathrm{i}} \\
\mathbf{k}_{\mathrm{p}}^{\prime} \\
\mathbf{k}_{\mathrm{i}}^{\prime}\end{array}$} & N/A & $0.54 \mathrm{rad} / \mathrm{sec}$ \\
\hline & & N/A & $3.83 \mathrm{rad} / \mathrm{sec}$ \\
\hline & & N/A & 0.73 \\
\hline & & N/A & 0.016 \\
\hline & & N/A & 1.07 \\
\hline & & N/A & 0.023 \\
\hline $\begin{array}{l}\text { Design } \\
\text { constraints }\end{array}$ & $\begin{array}{l}\text { Rigid-body velocity-loop gain margin } \\
\text { Rigid-body position-loop gain margin } \\
\text { Rigid-body velocity-loop phase margin } \\
\text { Rigid-body position-loop phase margin } \\
\text { Velocity-loop apparent gain margin al } 0.49 \mathrm{~Hz} \\
\text { Minimum rigid-body and controller damping ratio }\end{array}$ & $\begin{array}{l}\geq 6 \mathrm{~dB} \\
\geq 6 \mathrm{~dB} \\
\geq 45 \mathrm{deg} \\
\geq 45 \mathrm{dcg} \\
\geq 20 \mathrm{~dB} \\
\geq 0.5\end{array}$ & $\begin{array}{l}7.5 \mathrm{~dB} \\
7.6 \mathrm{~dB} \\
45.0 \mathrm{deg} \\
45.0 \mathrm{deg} \\
20.0 \mathrm{~dB} \\
0.51\end{array}$ \\
\hline
\end{tabular}


of the station is shown in Fig. 8. All the flexible modes below 5 $\mathrm{Hz}$ were incorporated in the simulation. An apparent gain margin of $20 \mathrm{~dB}$ and rigid-body gain and phase margins of $7.5 \mathrm{~dB}$ and $45 \mathrm{deg}$, respectively, are obtained as indicated in the figure.

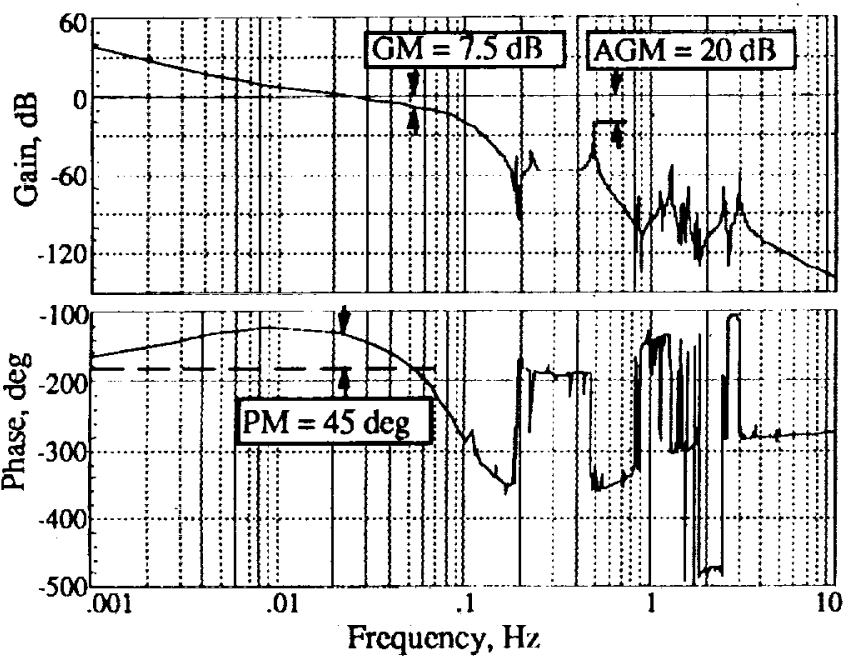

Fig. 8 Compensated Bode plot of port velocity open loop
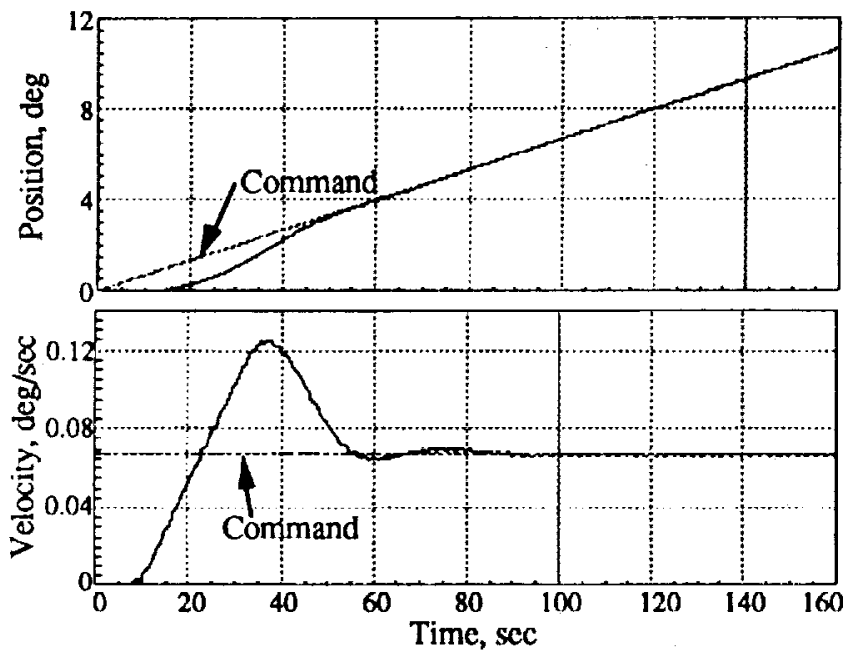

Fig. 9 Simulated position and velocity tracking responsc

The time response of the control system is simulated for a step velocity command of $4 \mathrm{deg} / \mathrm{min}$ and a ramp position command with a slope of $4 \mathrm{deg} / \mathrm{min}$. Figures 9 through 11 show the results of the simulation using all the flexible modes below 5 $\mathrm{Hz}$. The position command inpul and the resulting response are compared in Fig. 9. No motion occurs until the static friction is overcome. Then, after a bricf initial transient period, the tracking is performed accurately. The velocity response to the command is also shown in Fig. 9. The higher frequency component of the response corresponds to the flexible response of the structure at the dominant mode frequency. The flexible response is quite small and should cause no structural load problems. The position error, i.e., the difference between the position command and the actual position response, is shown in Fig. 10. The steady state pointing error, which should be less than $0.58 \mathrm{deg}$, is met within $40 \mathrm{sec}$. Jitter time history is also shown in Fig. 10. The steady-state jitter requirement is met in less than one minute.
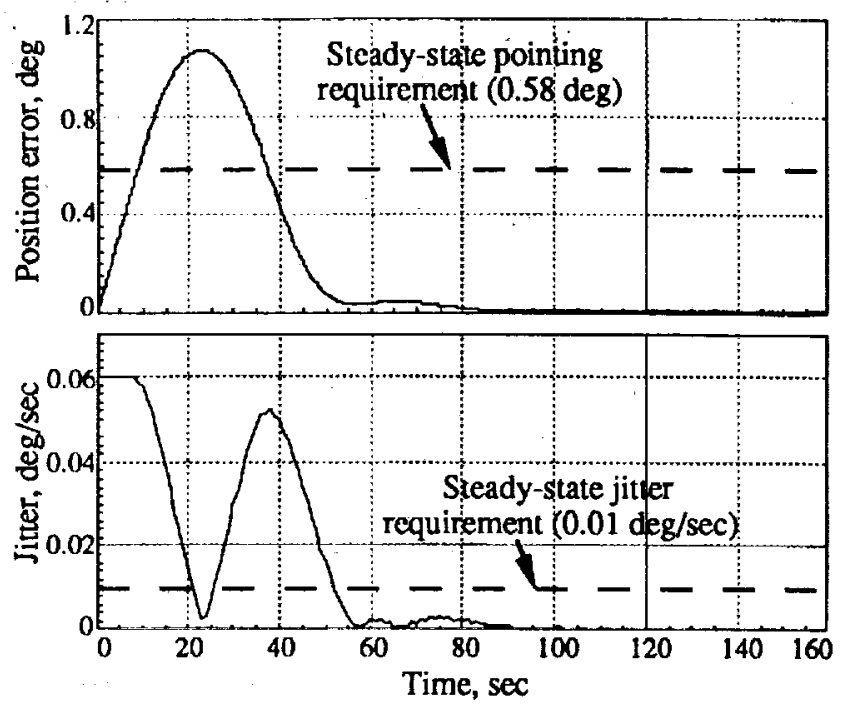

Fig. 10 Simulated position tracking error and jitter

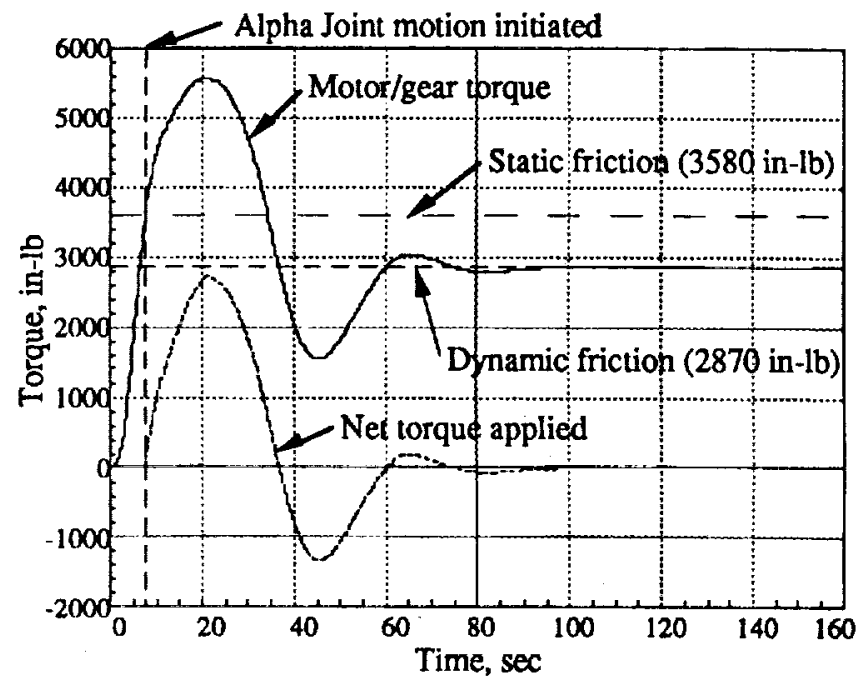

Fig. 11 Simulated motor/gear torque and net torque applied

Figure 11 illustrates the torque generated by the motor and gear train to perform the Alpha Joint pointing and the net torque applied to the station after overcoming the friction. No net torque is applied to the station until the torque overcomes the static friction. Once the motion of the Alpha Joint is initiated, the dynamic friction becomes effective and the magnitude of steadystate motor/gear torque is just enough to overcome the dynamic Iriction. As a result, the core structure of the station docs not experience a net torque applied from the joint motor and gear train after the initial transient period of $100 \mathrm{sec}$. The maximum torque available is approximately $30,000 \mathrm{in}-\mathrm{lbf}$. The peak motor torque required is well within the torque limit. Thereforc, the assumption that the motor is capable of producing the level of torque required by the synthesized design is verified. 


\section{Sensitivity of stability robustness and performance to structural narameter variations}

The nexible plant model used in the synthesis of design variables is characterized by its natural frequencies, mode shapes, and modal damping ratios. The bascline design variables presented in the previous section was obtained using "nominal" values of the modal parameters. These nominal modal parameters are analytical estimates and may be inaccurate. In order to address the effect of variation of the modal parameters on the control system performance, two types of sensitivity analyses are performed in this paper. The first study examines the change in the apparent gain margin (stability robustness measure) as the modal parameters vary while maintaining the baseline values of the design variables. In the second study, the values of the design variables are re-synthesized for the flexible plant with modificd modal parameters while enforcing the same design constraints as used for the baseline design. The resulting variations of the performance index and its components are investigated. For a set of fixed values of the design variables, the performance index is independent of the changes in structural modal parameters because the performance index is composed of the velocity and position loop bandwidths and the dominant rigidbody closed-loop pole. Thus, performance robusiness duc to structural parameter variation is not discussed in this paper.

\section{Sensitivity of stability robustness}

While keeping the baseline values of the design variables unchanged, the effect of modal parameter changes on the stability margin is examined. The gain and phase margins of the position and velocity loops and the locations of the rigid-body plant and controller poles remain unchanged since the values of the design variables and rigid body plant are fixed for this study. Only the apparent gain margin is influenced by the variation of the modal parameters. The baseline design parameters obtained for the nominal flexible plant provide $20 \mathrm{~dB}$ velocity-loop apparent gain margin at the frequencies of dominant modes. The position-loop always has a larger apparent gain margin. Therefore, this study examines only the change in the velocity-loop apparent gain margin as the modal paramieters of the dominant modes vary.

Figure 12 shows the sensitivity of stability margin to a variation in dominant mode frequencics and modal amplitudes while kecping the modal damping ratio constant at $0.1 \%$. Lines of constant apparent gain margins are shown in the figure. Changes in modal frequencies affect only the system A matrix. The modal amplitude is varicd by positive scalar multiplications of the mass nomalized mode shapes at the sensor and actuator locations simultancously. These affect the system $\mathbf{b}$ vector and $\mathbf{C}$ matrix. All modal parameter variations are represented in percentages.

As the dominant mode frequencies decrease, the resonant peaks of the two dominant modes move closer to the comer frequency of the bending filter $\left(\omega_{c}\right)$. Hence, the amount of rolloff at the dominant mode frequencies decreases resulting in a smaller apparent gain margin. This is indicated along the ordinate of Fig. 12. When the dominant mode frequencies are decreased by approximately $38 \%$ while kecping the modal amplitude constant, the apparent gain margin diminishes to zero and if the phase at this frequency is at -180 degrees the system becomes unstable. The origin corresponds to the nominal plant. It should also be noted that the robustness would be improved if the dominant mode frequencies are increased (not shown in Fig. 12). For a given frequency change, the reduction in robustness is secn along the abscissa of Fig. 12 as the modal amplitude increases. The increase in modal amplitude corresponds to the increase in height of the resonant peaks and hence reduces the apparent gain margin. A change of approximately $+210 \%$ in the modal amplitude of the dominant modes is required to nullify the apparent gain margin. A reduction in modal amplitude increases the apparent gain margin (not shown in Fig. 12).

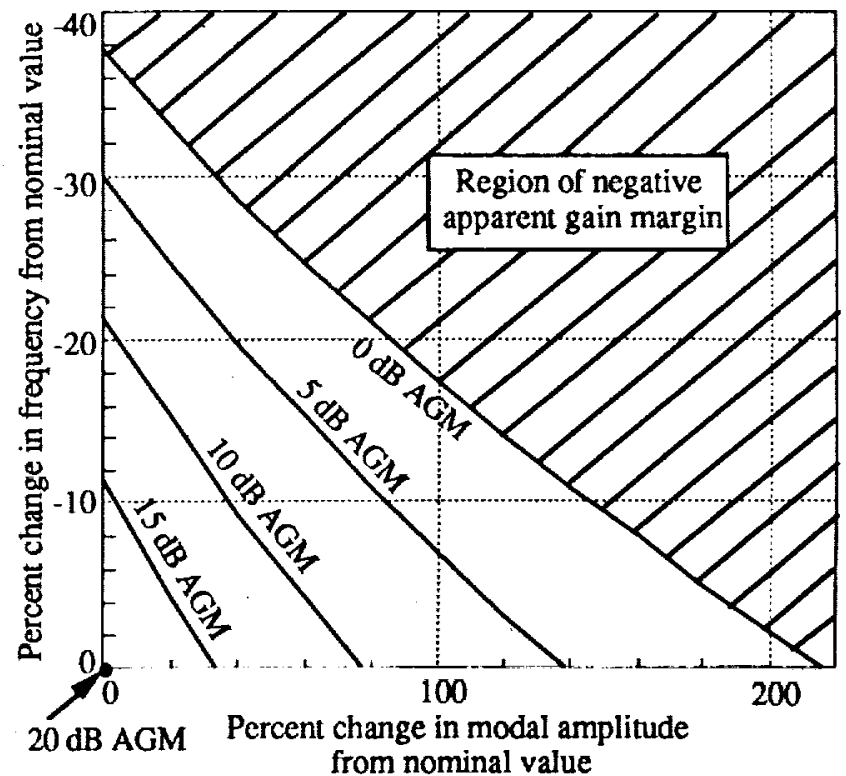

Fig. 12 Sensitivity of velocity-loop apparent gain margin to variations in frequency and modal amplitude of dominant elastic mode (modal damping $=0.1 \%$ )

Points interior to the axes correspond to combined errors in both modal frequencies and modal amplitudes. Regions of negative stability margins are also shown in Fig. 12. Space station structural parameters predicted analytically may contain apprcciablc errors. ${ }^{8}$ The inability of ground testing for model verification and the synthesis error of component modal characteristics are the major source of the errors. Thus, in order to compensate for the errors, the control system design should be robust to large variations in modal parameters. Figure 12 indicates that, for a nominal design using $20 \mathrm{~dB}$ apparent gain margin, the control system can tolerate a wide range of modal parameter variations.

Figure 13 depicts the sensitivity of stability robustness to a variation in modal damping ratio while keeping the dominant mode frequencies and modal amplitudes constant. The nominal plant has a conservative low damping of $0.1 \%$. As the modal damping ratio increascs, the resonant peak of the dominant modes decrease (the resonant peak is approximately proportional to $1 / 2 \zeta$ ). Thus, the apparent gain margin increases. As the modal damping ratio decreases the apparent gain margin decreases. 
However, this is not discussed due to the already conservative nominal value of modal damping ratio chosen.

\section{Sensitivity of control system performance}

In this study, the control system design variables are re synthesized as the modal parameters of the dominant modes vary. The constraints, as listed in Table 1, are enforced and the values. of the design variables are re-computed for each change in the value of a modal parameter to provide the most responsive tracking performance. The variation of the performance index and its three components is examined as the frequencies, modal amplitudes, and modal damping ratios change.

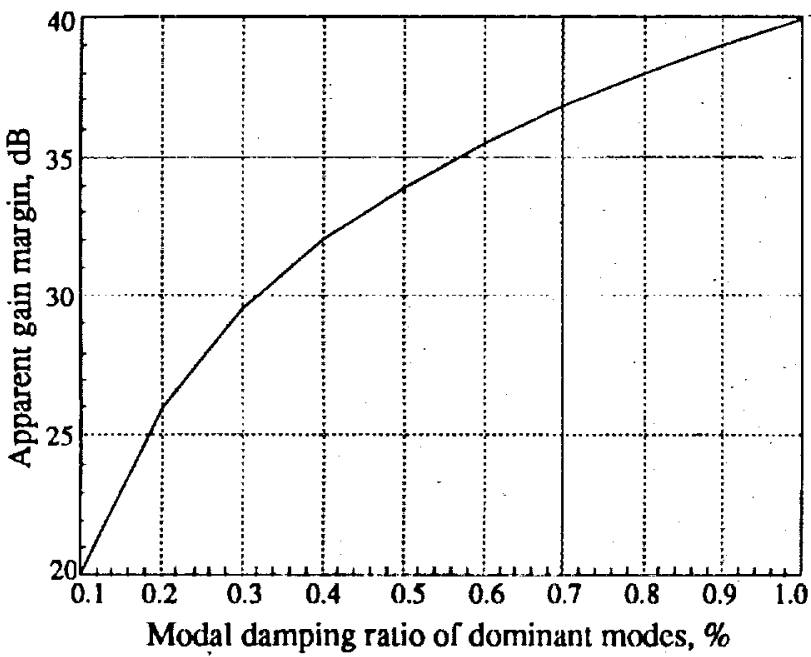

Fig. 13 Sensitivity of velocity-loop apparent gain margin to variations in modal damping ratio of dominant modes

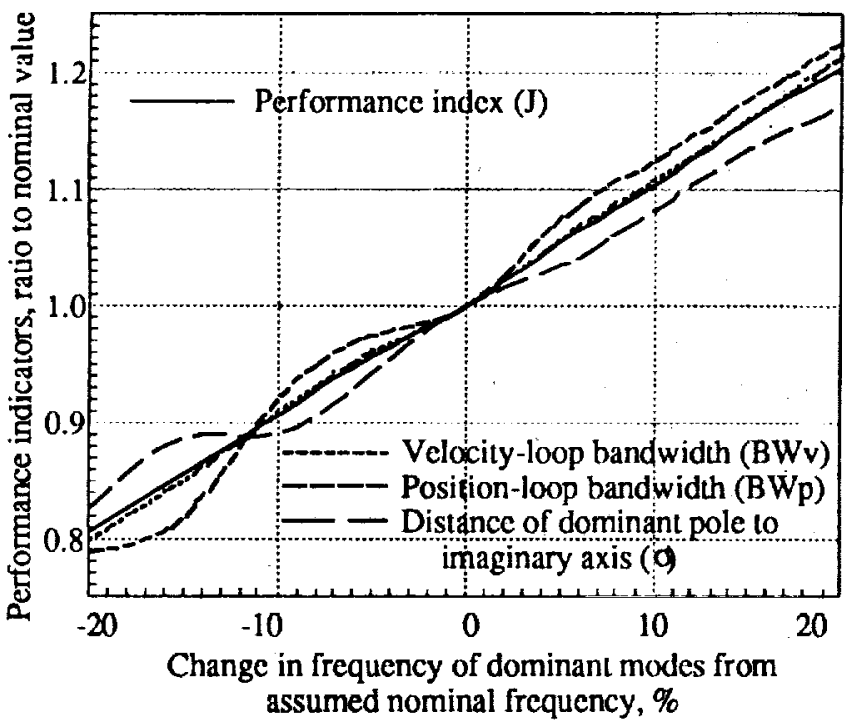

Fig. 14a Sensitivity of performance indicators to variation in frequency of dominant flexible modes

Figure 14a shows the variation of the performance indicators as the frequencies of the dominant modes change. The modal amplitudes and damping ratios are held at their nominal values. The ordinate is the ratio of the performance index $(J)$ and its indicators $\left(B W_{v}, B W_{p}\right.$, and $\sigma$ ) to their respective nominal values, The performance index consistently improves as the dominant mode frequencies increase and vice versa. As the dominant mode frequencies increase, the passband of the Butterworth filter can be extended. This results in an increase in the performance index and its components. The design variables are re-synthesized at the interval of $5 \%$ change in nominal frequency. For instance, the baseline design variables are used as an initial guess for the synthesis with $5 \%$ frequency change.

Figure $14 \mathrm{~b}$ illustrates the variation of the performance indicators as the modal amplitude of the dominant modes changes. The frequencics and modal damping ratios are fixed at their nominal values. The performance index and its components consistently decrease with increasing modal amplitude. As the resonant peaks of the dominant modes increase, the apparent gain margin constraint is violated. In order to meet the constraint, the corner frequency of the filter decreases while ensuring that the other constrains are not violated. Thus, the performance index and its components, in general, decrease. The design variables are re-synthesized at intervals of $10 \%$ change in nominal modal amplitude using the previous optimized design as a starting guess for design variables.

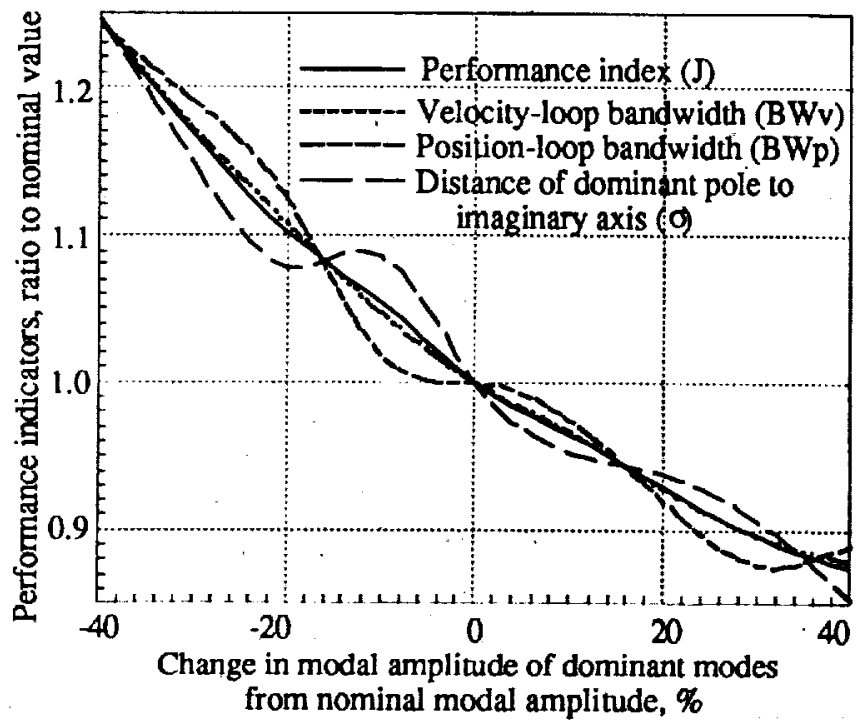

Fig. 14b Sensitivity of performance indicators to variation in modal amplitude of dominant flexible modes

The variations in the performance indicators with respect to the changes in modal damping ratios are shown in Fig. 14c. As the modal damping ratio increases, the resonant peaks of the dominant flexible modes reduces and the apparent gain margin constraint becomes less critical. The passband of the low-pass filter may be extended, increasing the bandwidth until the apparent gain margin constraints or the other constraints become active. Hence the performance indicators should increase or at worst rcmain constant. Figure $14 \mathrm{c}$ indicates that the performance incrcases until the modal damping ratio is raised to approximately $0.4 \%$. At this value, the apparent gain margin constraint is no longer active and the performance indicators remain constant for additional increase in damping. Thus, attempts to increase the modal damping ratio would be beneficial in increasing control system performance only up to a certain 
level. The magnitude of modal damping ratio at which this performance saturation occurs will depend on the constraints imposed.

The time domain analysis for each set of optimized values of the design variables should be performed to check whether the sicady-state pointing and jitter requirements are satisfied. The lime domain response will depend on the values of the design variables but it will damp out since the closed-loop rigid-body pole constraint ensures a minimum damping ratio of 0.5 . The steady-state tracking errors could be met by adjusting the integrator limits in the controllers.

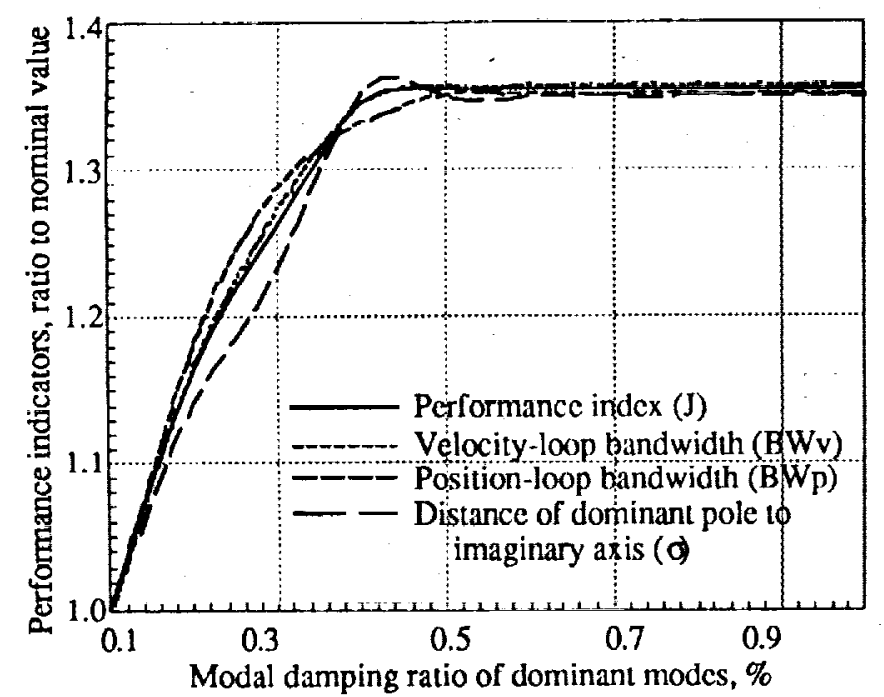

Fig. 14c Sensitivity of performance indicators to variation in modal damping ratio of dominant flexible modes

\section{Conclusions}

The significant components of the space station related to the Alpha Joint control were described and the photovoltaic array sun tracking control system was presented. A baseline design was determined using constrained optimization techniques to mect design requirements, to provide a given level of stability robustness, and to obtain the most responsive tracking capable consistent with the assumed structural characteristics. Performance of the baseline design was discussed and the sensitivity of the stability margin was examined for variations in the natural frequencies, mode shapes and damping ratios of dominant structural modes.

The dominant elastic modes occurred approximately at 0.5 $\mathrm{Hz}$ and were well below the maximum Alpha Joint controller closed-loop bandwidth of one $\mathrm{Hz}$ specified in the design requirements. These modes exhibited symmetric and antisymmetric photovoltaic array bending coupled with rigid rotation of the transverse booms outboard of the Alpha Joint. The highest bandwidth achicvable for a design which met all requirements for phase and gain stability robustncss, steady state bchavior and reasonable settling time was $0.054 \mathrm{~Hz}$. for the velocity loop, approximately a decade below the dominant mode frequencies. The design provided enough robustness to tolerate a sizeable error of up to 40 percent in the predicted resonant frequency of the dominant modes and to tolcrate an error in the predicted modal amplitude of up to 200 percent. The station will not be tested as a complete system before being placed in orbit and modal frequencies will have to be predicted using information from component tests and unvalidated analytical models. Hence, the high levels of stability margin suggested in this study for the nominal design seem appropriate.

The paper concludes with an investigation on the sensitivity of performance indicators as the modal parameters of the dominant modes vary. The design variables are re-synthesized for varying modal parameters in order to achieve the most responsive tracking performance while satisfying the design requirements. This procedure of re-optimizing design parameters would be useful in improving the control system performance if accurate modal data are provided through an onorbit modal identification experiment.

\section{References}

1. Laskin, R. A, Estus, J. M., Lin, Y. H., Spanos, J. T., and Scatter, C. M., "NASA Office of Space Science and Applications Study on Space Station Attached Payload Pointing," AlAA Paper 88-4105, Proceedings of AIAA Guidance, Navigation and Control Conference, Minncapolis, MN, Aug. 1988, pp. $430-443$. 2. Merritt, L. V., "Rotary Joint Servo Loops: General Description with Performance Models," Rough Draft of EM-2ME-62, Lockheed Missiles \& Space Company, Space Station Work Package 2, March 1990.

3. Merritt, L. V., "The Space Station Rotary Joint Motor Controller," Motion Journal, January/February 1990, pp. 14-22.

4. Bone, J. and Nuttall, N., "Performance Assessment of the Solar Alpha Rotary Joint Control System with a Flexible Dynamic Load Model," EM-2-ME-014, PDR document MDC H6506, Space Station Work Package 2, June 1990.

5. Lam, H. Y-F., Analog and Digital Filters: Design and Realization, Prentice Hall, 1979, p. 301.

6. MATRIXx Optimization Module Manual, Edition 2, Integrated Systems, Inc., October 1989.

7. Ye, Y. Y., Ph.D. Dissertation, Department of EngineeringEconomic Systems, Stanford University, 1987.

8. Hanks, B. R., "Dynamic Challenges for 21st-Century Spacecraft," Keynote Address, 21st Shock and Vibration Symposium, Pasadena, CA, October 1990. 
NASA TM - 104153

4. Title and Subtitle

Sensitivity of space Station Alpha Joint Robust Controller to Structural Modal Parameter Variations

August 1991

6. Performing Organization Code

\section{Author(s)}

8. Performing Organization Repon No.

Renjith R. Kumar, Paul A. Cooper, and Tae W. Lim

\section{Performing Organization Name and Address \\ NASA Langley Research Center \\ Hampton, VA 23665-5225}

\section{Sponsoring Agency Name and Address}

National Aeronautics and Space Administration Washington, DC 20456-0001
10. Work Unit No.

$590-14-31-02$

11. Contract or Grant No.

13. Type of Repon and Period Covered

Technical Memorandum

14. Sponsoring Agency Code

\section{Supplementary Notes}

Presented at the AIAA Guidance, Navigation and Control Conference, August 12-14, 1991 Renjith R. Kumar:Analytical Mechanics Associates, Inc., Hampton, Virginia; Paul A.Cooper: NASA Langley Research Center, Hampton, Virginia; Tae W. Lim:Lockheed Engineering and Sciences Company, Hampton, Virginia

\section{Abatract}

The photovoltaic array sun tracking control system of space station Freedom is described in this paper. A synthesis procedure for determining optimized values of the design variables of the control system is developed using a constrained optimization technique. The synthesis is performed to provide a given level of stability margin, to achieve the most responsive tracking performance, and to meet other design requirements. Performance of the baseline design, which is synthesized using predicted structural characteristics, is discussed and the sensitivity of the siacility margin is examined for variations of the frequencies, mode shapes and damping ratios of dominant structural modes. The design provides enough robustness to tolerate a sizeable error in the predicted modal parameters. This paper concludes with an investigation on the sensitivity of performance indicators as the modal parameters of the dominant modes vary. The design variables are re-synthesized for varying modal parameters in order to achieve the most responsive tracking performance while satisfying the design requirements. This procedure of re-optimizing design parameters would be useful in improving the control system performance if accurate modal data are provided through an on-orbit modal ID expt.

17. Key Words (Suggested by Author(s))

Space Station Freedom Control/Structure Interaction Tracking Control Stability Sensitivity

\section{Distribution Stotoment}

Unclassified--Unlimited

Subject Category 39
19. Security Classif. Iof this report)

Unclassified
20. Security Classif. (ot this pagel

Unclassified
21. No. of paces

22. Price

11 $\mathrm{AO} 3$ 
